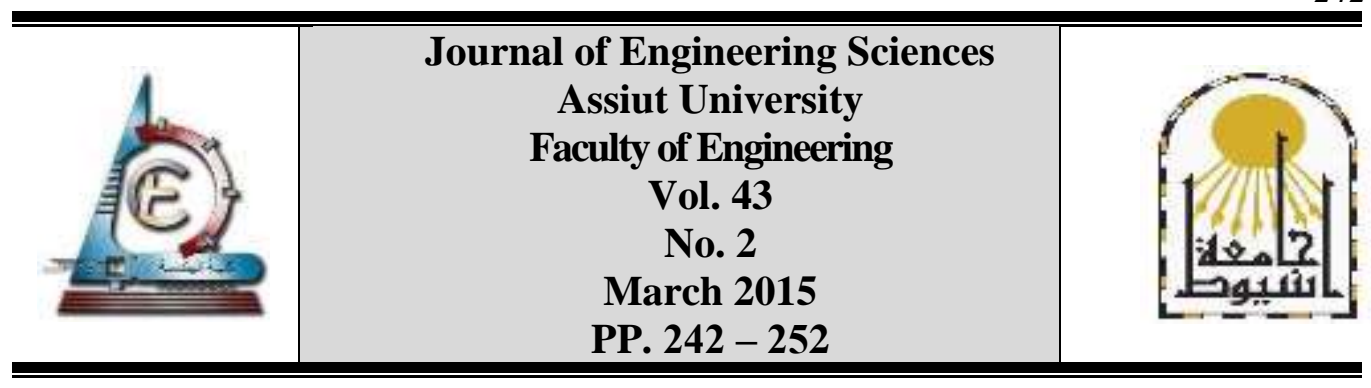

\title{
ALL OPTICAL OVSF CODE GENERATOR REALIZATION USING OPTICAL FLIP FLOP AND OPTICAL HARDLIMITTERS
}

\author{
Tamer A. Moniem * and Noha EL Mosilhy \\ Canadian International College CIC, New Cairo, Egypt \\ Noha_elmosilhy@cic-cairo.com
}

(Received 7 March 2015; Accepted 22 April 2015)

\begin{abstract}
The orthogonal variables spreading factor (OVSF) provides variables data rates to flexible support many applications with different bandwidth. This paper presents an optical OVSF code generator using all optical gates and a set of all optical flip-flops based on two coupled polarization switches (PSWs). The optical OVSF code generator helps to use the OVSF code technique in optical communications. The general objective is to make the OVSF code tree suitable to support a lot of users in the optical communication links. The overall design and the results are discussed through the realization and the computing numerical simulation to confirm its operation and feasibility. The proposed scheme has been theoretically demonstrated for a spreading factor of 4 and 8 .
\end{abstract}

Keywords: Optical Flip Flops, Optical Gates, Bragg Grating, OVSF, SOA.

\section{Introduction}

The Modern Code Division Multiple Access (CDMA) uses the OVSF technology codes to provide multiuser access. It has a lot of uses in different applications mainly with digital communications and test pattern generations.

In the OVSF, a single code using one transceiver can support higher and variable data rates with less complexity than the multi-code orthogonal constant spreading factor (OCSF) [1]. The OVSF techniques of code placement and allocation in CDMA guarantee the orthogonally between all users in different communication channels.

The emergence of increasingly high speed and the number of users in optical communication systems demands the OVSF codes technique in the optical communication links. The performing of signal processing operations entirely within the optical domain would exploit the speed and parallelism inherent to optics [2].

The overall optical design of linear feedback shift register (LFSR) of pseudorandom binary sequence (PRBS) generator of CDMA using the hardlimiter and a set of series all

\footnotetext{
* Corresponding author.

E-mail address: tamer_abdelrahman@cic-cairo.com
} 
optical D flip flops has been demonstrated by Tanay and Tamer et al. (2013) [3]. Zoirios et al. (2011) [4] have demonstrated an all optical pseudorandom generators using the Terahertz optical asymmetric Demultiplexer (TOAD).

In this paper, the basic operation and design of all-optical OVSF code generator of the modern CDMA used in Universal Mobile Telecommunication System (UMTS) standard are proposed. The overall optical design in the optical communication system eliminates the conversion from optical to electrical and vice versa and reduces the latency due to this conversion [3].

An overview of the OVSF codes and its code placement methods are presented in Section 2. The digital electronic circuit of OVSF code is represented in Section 3. The building construction and operation of optical devices such as all optical gates and all optical flip flops are proposed in Section 4. Finally, the overall optical design of OVSF code generator and the results output from this generator are displayed in Section 5.

\section{OVSF codes in the modern CDMA}

The CDMA system allows multiple users to transmit at the same time in the same frequency band [3]. The CDMA suffers from the high blocking probability because the number of orthogonal codes is limited. There are two steps applied to users in a CDMA system. The first step is transforming every bit into a code sequence, where the code sequence has a length called the spreading factor (SF). The second step is called the scrambling process, where the scrambling codes are used to separate the signal from different sources $[5,6]$. The OVSF in CDMA achieves a flexible support of mixed and variable data rates at the same bandwidth, where the OVSF codes are used as the channelization codes [7].

The OVSF codes can be represented and defined by the code tree shown in Figure 1. Each OVSF code can be presented by a channelization code $\mathrm{C}_{S F, N}$, where $S F$ is the spreading factor in the range $[4-512]=\left[2^{2}-2^{9}\right]$ and $N$ is the branch identification number of user, $1<N<(S F-1)$. The number of codes at each level is equal to the value of SF. All codes in the same layer are orthogonal, while codes in different layers are orthogonal if they do not have an ancestordescendant relationship such as $\mathrm{C}_{4,2}$ and $\mathrm{C}_{2,2}$. Leaf codes have the minimum data rate, which is denoted by $1 \mathrm{R}$ and the data rate is doubled whenever we go one level up the tree [8].

8R

$4 R$

2R

R

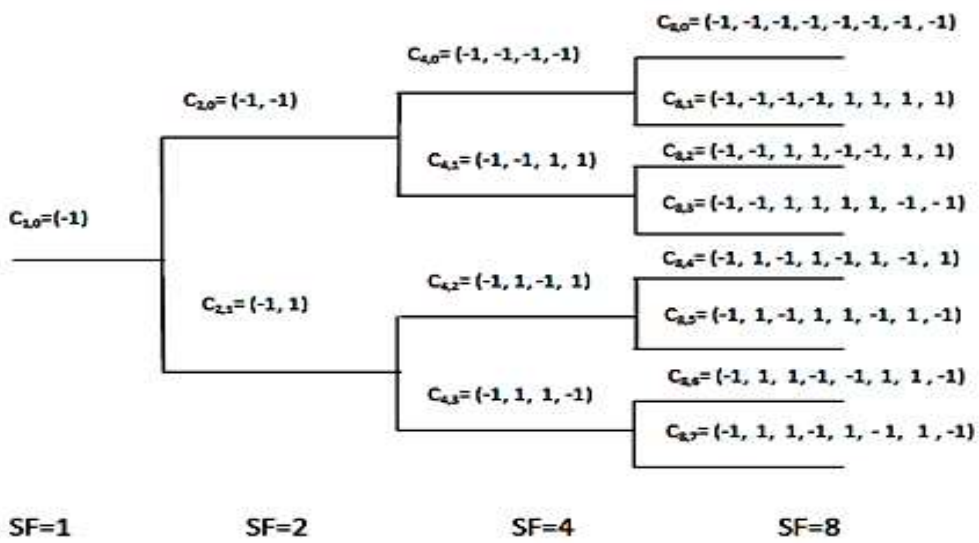

Fig. 1. Tree of OVSF codes till spreading factor $\mathrm{SF}=8$ 
There are many techniques used for code placement, where the code placement techniue is allocation policy for a new user requesting for a code of rate $k \mathrm{R}$. There are two possible prbabilties that occur at no free codes exists, the first one is rejecting this call (i.e. Code Blocking) and the second is to relocate the existing codes used for a new user [8]. The OVSF code placement has been investigated as follows. Chao et al. [8] proposed a simple algorithm based on single and multi- codes placement and replacement schemes, but it possibly incurs many fragmented codes which produce a code blocking problem. The proposed strategies in Chao [8] and Chen et al. [10] algorithms are based on three strategies of Random, Leftmost and Crowded -first. The OVSF code placement is assigned for IMT-2000 for multi-rate with less complexity by Cheng and Lin [9]. Recently, Chen and Lung [6] proposed un-sequence property of linear-code chains to design a new code placement and replacement mechanism by identifying a linear-code chains (LCCs) and nonlinear-code trees (NCTs) in the Rotated OVSF code tree. The logic circuit of OVSF code tree generator was designed by Dong [11] and Boris [12].

\section{The digital logic circuit of OVSF codes generator}

This section represents the electrical logic circuit of OVSF codes generator. Boris et al. [12] proposed a logic circuit for OVSF code generator. The hardware of the OVSF codes generator consists of a set of logic gates and T flip flops. The chip sequence is specified in binary number set $\{-1,+1\}$ for digital logic operating in the set $\{0,1\}$. The OVSF codes generator is shown in Figure 2 [12]. This generator consists of 9 bits OVSF code ID register $\left(\mathrm{n}_{8}-\mathrm{n}_{0}\right)$, this ID register represent the identification number of user $\mathrm{N}$, supposing the channelization code $\mathrm{C}_{8,6}$ is required

$\mathrm{N}=6=\mathrm{n}_{3} \mathrm{n}_{2} \mathrm{n}_{1} \mathrm{n}_{0}=0110$

$S F=8$

Also, the OVSF generator contains a set of AND gates, XOR gates, and 8 bits binary ripple counter as shown in Figure 2. That code generator is capable of producing codes with a spreading factor over range $S F=4$ to 512 [12]. The logical expression of OVSF code can be expressed as:

OVSF Code $=\left(n_{0} \cap b_{8}\right) \oplus\left(n_{1} \cap b_{7}\right) \oplus\left(n_{2} \cap b_{6}\right) \oplus\left(n_{3} \cap b_{5}\right) \oplus\left(n_{4} \cap b_{4}\right) \oplus$ $\left(n_{5} \cap b_{3}\right) \oplus\left(n_{6} \cap b_{2}\right) \oplus\left(n_{7} \cap b_{1}\right) \oplus\left(n_{8} \cap b_{0}\right)$

$\oplus$ and $\cap$ indicates the XOR and AND operation, respectively.

The design counter is counting incrementally from 0 to $S F-1$, where $b_{8}$ is always the most significant bit (MSB), and the least significant bit (LSB) is specified by the variable spreading factor $S F$. The MSB of ID code number N is always enabled by the LSB of the counter [12]. In this case, a regular binary ripple counter is required to count between 0 and $S F-1$. Based on the logic circuit of OVSF code generator, the code generated for all channelization codes in the level of $\mathrm{SF}=8$ for binary counting $\mathrm{b}_{8} \mathrm{~b}_{7} \mathrm{~b}_{6}$ is depicted in Table 1 . The logic circuit of binary ripple counter is depicted in Figure 3 [12]. The counter consists of a series T flip flops of 9 bits. The LSB counting is controlled by $S F$ register as depicted in Table 2.

The MSB of counter is always $\mathrm{n}_{8}$, and the LSB is controlled by SF register code. The stored value in SF register represents the information describing the spreading factor and the code ID required to generating the code. 
OVSF Code ID Register

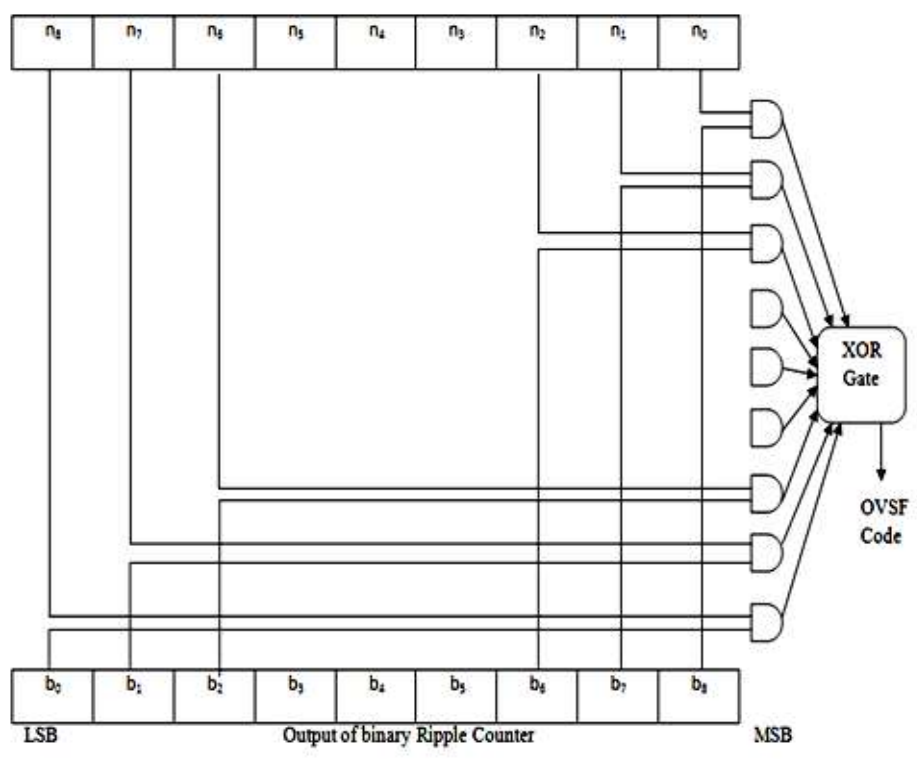

Fig. 2. The logic circuit of OVSF codes generator [12].

Table1.

Generation of channelization code for $S F=8$

\begin{tabular}{|c|c|c|c|c|c|c|c|c|}
\hline $\begin{array}{c}\text { Counter Code } \\
\text { O/P } \\
\text { bsbobo }\end{array}$ & 000 & 001 & 010 & 011 & 100 & 101 & 110 & 111 \\
\hline Logic Operation & \multirow[t]{2}{*}{$n_{3}$} & \multirow[t]{2}{*}{$n_{3 \oplus} n_{2}$} & \multirow[t]{2}{*}{$n_{3 \oplus} n_{1}$} & \multirow[t]{2}{*}{$n_{3 \oplus} n_{2 \oplus} n_{1}$} & \multirow[t]{2}{*}{$n_{3 \oplus} n_{0}$} & \multirow[t]{2}{*}{$n_{3 \oplus} n_{2 \oplus} n_{0}$} & \multirow[t]{2}{*}{$n_{3 \oplus} n_{1 \oplus} n_{0}$} & \multirow[t]{2}{*}{$n_{3 \oplus} n_{2 \oplus} n_{1 \oplus} n_{0}$} \\
\hline OVSF Code & & & & & & & & \\
\hline $\begin{array}{l}\mathrm{C}_{8,0} \\
\left(\mathrm{n}_{3 \mathrm{n}, \mathrm{n} ! n}=0000\right)\end{array}$ & 0 & 0 & 0 & 0 & 0 & 0 & 0 & 0 \\
\hline $\begin{array}{l}\mathrm{C}_{8,1} \\
\left(\mathrm{n} 3 \mathrm{n}, \mathrm{n} ! n_{1}=0001\right)\end{array}$ & 0 & 0 & 0 & 0 & 1 & 1 & 1 & 1 \\
\hline $\begin{array}{l}\mathrm{C}_{8,2} \\
\left(\mathrm{n}_{3 \mathrm{n}, 2 n ! n}=0010\right)\end{array}$ & 0 & 0 & 1 & 1 & 0 & 0 & 1 & 1 \\
\hline $\begin{array}{l}\mathrm{C}_{8,3} \\
\left(\mathrm{n}_{3} \mathrm{n}_{2} \mathrm{n}_{1} \mathrm{n}=0011\right)\end{array}$ & 0 & 0 & 1 & 1 & 1 & 1 & 0 & 0 \\
\hline $\begin{array}{l}\mathrm{C}_{8,4} \\
\left(\mathrm{n}_{3} \mathrm{n}_{2} \mathrm{n}_{1} \mathrm{n}=0100\right)\end{array}$ & 0 & 1 & 0 & 1 & 0 & 1 & 0 & 1 \\
\hline $\begin{array}{l}\mathrm{C}_{8,5} \\
\left(\mathrm{n} 3 \mathrm{n}_{2} \mathrm{n}_{1} \mathrm{n}=0101\right)\end{array}$ & 0 & 1 & 0 & 1 & 1 & 0 & 1 & 0 \\
\hline $\begin{array}{l}\mathrm{C}_{8,6} \\
\left(\mathrm{n}_{3} \mathrm{n}_{2 n ! n}=0110\right)\end{array}$ & 0 & 1 & 1 & 0 & 0 & 1 & 1 & 0 \\
\hline $\begin{array}{l}\mathrm{C}_{8,7} \\
\left(\mathrm{n}_{3} \mathrm{n}_{2} \mathrm{n}_{1} \mathrm{n}=0111\right)\end{array}$ & 0 & 1 & 1 & 0 & 1 & 0 & 0 & 1 \\
\hline
\end{tabular}




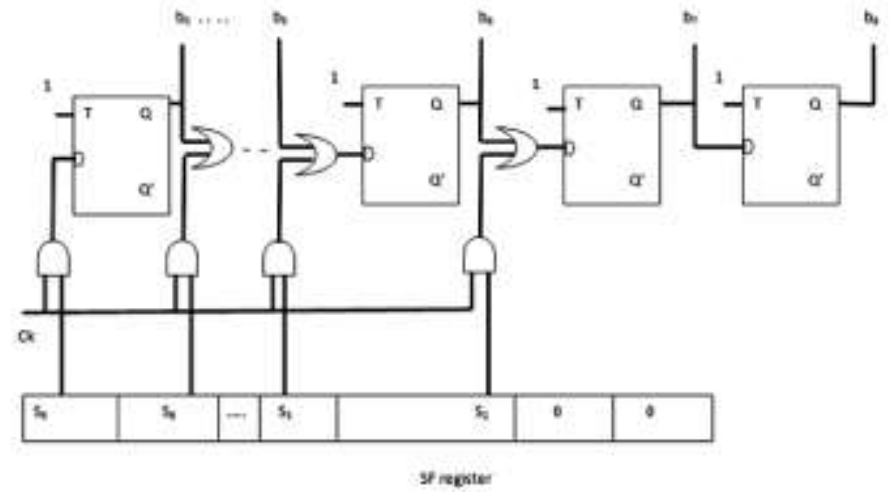

Fig. 3. The logic circuit of 9 bits ripple counter with variable location of the LSB [12]

Table 2.

The contents of $S F$ register for different spreading factors

\begin{tabular}{|c|c|c|}
\hline SF & $\begin{array}{c}\text { SF Register } \\
S_{9} S_{8} S_{7} S_{6} S_{5} S_{4} S_{3} S_{2} S_{1} S_{0}\end{array}$ & Counter Range \\
\hline 1 & 0000000000 & 0 \\
\hline 2 & 0000000000 & 0 \\
\hline 4 & 0000000100 & $0-4$ \\
\hline 8 & 0000001000 & $0-8$ \\
\hline 32 & 0000010000 & $0-16$ \\
\hline 64 & 0000100000 & $0-32$ \\
\hline 128 & 0001000000 & $0-64$ \\
\hline 256 & 0010000000 & $0-128$ \\
\hline 512 & 0100000000 & $0-256$ \\
\hline
\end{tabular}

\section{All optical hardware components}

This section represents the optical components used in the realization of the proposed optical OVSF generator.

\subsection{All optical AND, OR and XOR gates}

Figure $4[3,13]$ illustrates an all-optical XOR and AND gates using the Bragg grating as a hardlimiter. Inputs A and B get combined into a single beam. The transmitted intensity is defined as $\mathrm{O} 1$ output and the reflected value as output $\mathrm{O} 2$. We bias the hardlimiter at a limiting value $\mathrm{a}=2$. If one of the inputs is 0 and the other 1 , the output at $\mathrm{O} 1$ is 0 and at $\mathrm{O} 2$ is 1 . If both inputs $A$ and $B$ have the value of 1 , then 2 is transmitted and 0 reflected. If both $\mathrm{A}$ and $\mathrm{B}$ inputs have the value of 0 , then 0 is transmitted and a 0 is reflected. Thus, O1 yields the result of an AND operation and $\mathrm{O} 2$ the result of a digital XOR. The intensity value 2 output of O1 (AND) must be normalized to yield a digital output [13,14].

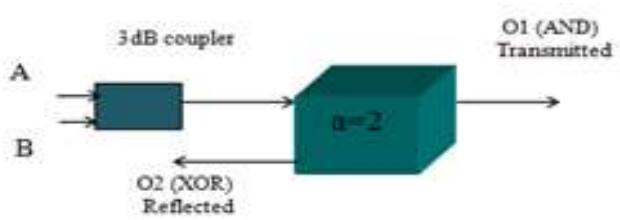

Fig. 4. All optical XOR and AND gates [3, 13]. 
Figure 5 [13] illustrates the use of the Bragg grating as a hardlimiter in the construction of OR gate. Inputs A and B are first combined into a single beam. The transmitted intensity is defined as the $\mathrm{O} 1$ output and the reflected value as the $\mathrm{O} 2$. We bias the hardlimiter at a limiting value $\mathrm{a}=1$. If one of the inputs is 0 and the other 1 , the output at $\mathrm{O} 1$ is 1 and at $\mathrm{O} 2$ is 0 . If both inputs $A$ and $B$ have the value of 1 , a 1 is transmitted and 1 reflected. Thus, O1 yields the result of an OR operation.

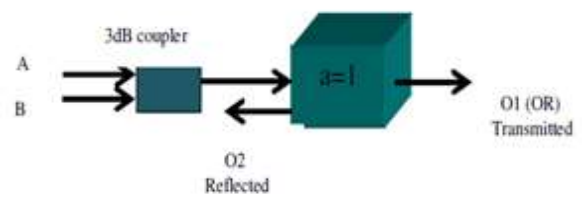

Fig. 5. All Optical OR gate [13].

\subsection{All optical JK flip flop}

The structure of the all-optical J-K flip-flop memory is depicted in Figure 6. It is consists of all optical flip flop based on two coupled polarization switches $[2,14]$ and a set of all optical logic gates based on the hardlimiters that are added to convert it into JK flip flop [15]. The first PSW1 outputs light that is injected into the second PSW2. Hence, the light that PSW1 outputs acts as a saturating control signal for SOA2 that can suppress PSW2 and the light that PSW2 outputs can act as a saturating control signal for the SOA1 to suppress PSW1. The optical pulses are injected into PSW1 via Port I of PBS (polarization beam splitter) to set the flip-flop in State1. The optical pulses are injected into PSW2 via Port II of PBS to reset the flip-flop in State 2 [14]. The hardlimiters shown in Figure 5 are biased at a limiting value $a=3$ to obtain optical AND gates with three inputs [15]. The output Q is applied to the AND gate with K terminal and clock pulse CK inputs. The flip-flop is cleared during a clock pulse if Q was previously 1. Similarly, output Q' is applied to AND gate with J terminal and clock (CK) inputs, and the flip-flop is set with a clock pulse if Q' was previously 1 . Inputs $\mathrm{J}$ and $\mathrm{K}$ behave like inputs $\mathrm{S}$ and $\mathrm{R}$ to set and clear the flip flop [14]. The T flip flop can be obtained from a J-K type if both inputs are tied together [16]. The truth table of J-K flip flops is depicted in Table 3. The logical expression of the inputs Set $(\mathrm{S})$ and Reset $(\mathrm{R})$ can be expressed as:

$$
\begin{aligned}
& S=Q_{n}^{\prime} \cap J \cap C k \\
& R=Q_{n} \cap K \cap C k
\end{aligned}
$$

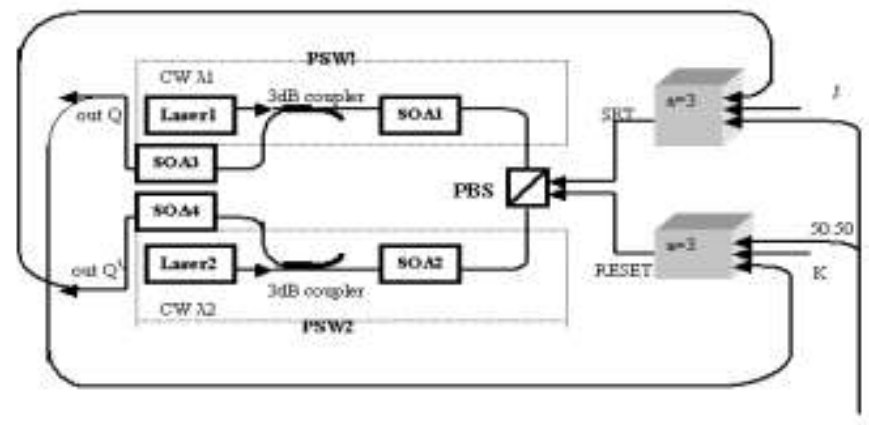

CK.

Fig. 6. All optical J-K flip flop based on two coupled polarization switches and optical gates. 
Table 3.

The truth table of JK flip flop

\begin{tabular}{|c|c|c|c|}
\hline$J$ & $K$ & $Q_{n+1}$ & $Q^{\prime}{ }_{n+1}$ \\
\hline 0 & 0 & $Q_{n}$ & $Q^{\prime}{ }_{n}$ \\
\hline 0 & 1 & 0 & 1 \\
\hline 1 & 0 & 1 & 0 \\
\hline 1 & 1 & $Q_{n}^{\prime}$ & $Q_{n}$ \\
\hline
\end{tabular}

\section{All optical implementation of OVSF code generator}

This section introduces the design of overall optical implementation, experimental results, and the simulation analysis of the OVSF generator. The design of generator is based on the logic design depicted in Figure 2 and 3 by using the proposed optical components in Section 4. The performance and accuracy of the design of the optical circuit is evaluated through experimental and numerical simulation to confirm its feasibility in terms of the choice of the critical parameters.

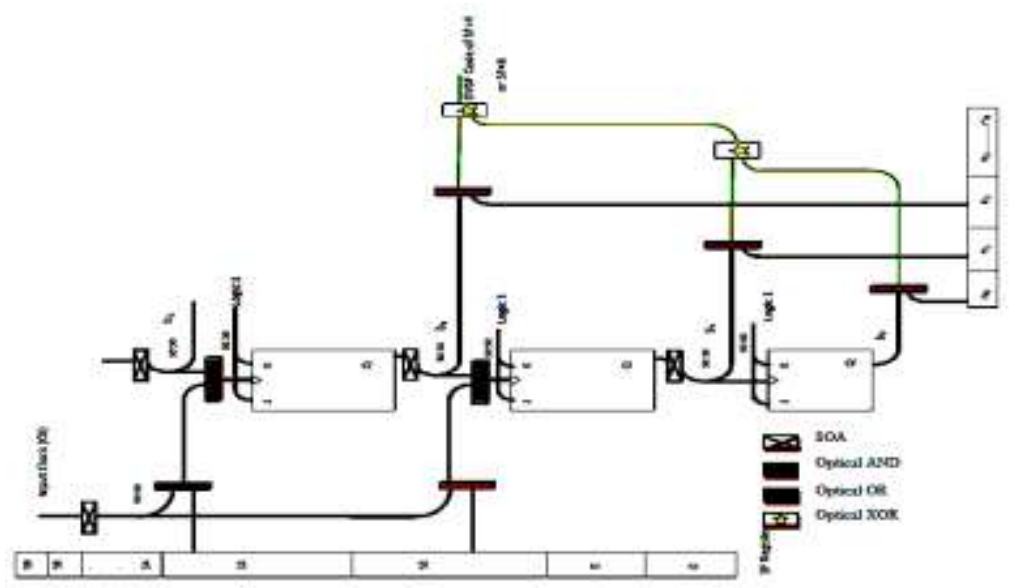

Fig. 7. The overall all optical OVSF code generator including the ripple counter of T flip flops, SF register, and optical gates

Figure 7 shows a section of the circuit diagram of all-optical OVSF code generator, it consists of a cascaded all-optical JK flip flops, and a set of optical hardlimiter AND gates, OR gates, and XOR gates which is equivalent to modulo 2 adder. The two inputs $\mathrm{J}$ and $\mathrm{K}$ of each flip flop are tied together to obtain a $\mathrm{T}$ flip flop, and this common input is set by logic input 1 .

SOA's (semiconductor optical amplifier) are used to compensate the losses of the cascaded connections between flip flops and optical couplers of 50:50 by adjusting the SOA current injection from $40 \mathrm{~mA}$ to $60 \mathrm{~mA}$ for different SOAs [17]. This injected current is used to control on the gain of SOA.

In order to calibrate and validate the model, experimental results were used. The theoretical parameters were heuristically refined until the best agreement between simulation and experiment, and the values are presented in Table 4, for a central wavelength of $1550 \mathrm{~nm}$. 
Table 4.

The theoretical parameters of SOA

\begin{tabular}{|c|c|c|}
\hline Parameter & Definition & Value \\
\hline $\mathrm{w}$ & Cavity width & $1.35 \mu \mathrm{m}$ \\
\hline $\mathrm{d}$ & Cavity height & $0.21 \mu \mathrm{m}$ \\
\hline $\mathrm{l}$ & Cavity length & $670 \mu \mathrm{m}$ \\
\hline $\mathrm{a}$ & Transversal section gain & $1.29 \times 10^{-16} \mathrm{~cm}^{2}$ \\
\hline $\mathrm{N}_{\mathrm{tr}}$ & Transparency carrier dens. & $2.1 \times 10^{18} \mathrm{~cm}^{-3}$ \\
\hline$\alpha$ & Attenuation & $2100 / \mathrm{m}^{2}$ \\
\hline $\mathrm{R}$ & Facet reflection & 0.0001 \\
\hline$\beta$ & Line-width enhancement factor & 5 \\
\hline $\mathrm{n}_{\mathrm{ef}}$ & Effective refractive index & 3.414 \\
\hline$\alpha_{\text {ins }}$ & Total insertion loss & $6.3 \mathrm{~dB}$ \\
\hline $\mathrm{E}_{\mathrm{sat}}$ & Saturation energy & $1.25 \mathrm{pJ}$ \\
\hline$\Gamma$ & Confinement factor & 0.39 \\
\hline
\end{tabular}

The outputs of SF register are applied to optical ANDs with the external clock to control the counting of optical counter according to Table 2. The optical counter consists of a series of optical T flip flops based on two coupled polarization switches which are presented in Section 4.2. The output of counter is optically AND with the identification code N. The outputs of all optical ANDs are applied to XOR gates according to Eq.(1) to finally obtain the OVSF code.

The OVSF code is numerically simulated using Beam Prop method V.7 and Optowave simulation program V.2. The wavelengths of each flip-flop are $1550 \mathrm{~nm}$ for $\lambda_{1}$ and 1552 $\mathrm{nm}$ for $\lambda_{2}$. There is an external light input at wavelength of $1600 \mathrm{~nm}\left(\lambda_{3}\right)$ which is injected as a clock pulse (CK). The optical clock pulses are injected approximately at every $0.1 \mathrm{~ns}$ with clock speed of $9-10 \mathrm{GHz}$ with duration equal to $0.05 \mathrm{~ns}$.

Figure 8 shows the result backed by the simulation results of optical OVSF generator $\mathrm{C}_{8,6}$ at $\mathrm{SF}=8$, where the identification number is $\mathrm{N}=6=\mathrm{n}_{3} \mathrm{n}_{2} \mathrm{n}_{1} \mathrm{n}_{0}=0110$ and the SF register code is adjusted at 0000001000 . At $S F=8$, the counter is counting incrementally from 0 to 8 , where $b_{8}$ is always the MSB, and the LSB is $b_{6}$ as shown in Figure 8. The logic expression of OVSF code at this case can be expressed as:

$$
\text { OVSF Code }=\left(n_{0} \cap b_{8}\right) \oplus\left(n_{1} \cap b_{7}\right) \oplus\left(n_{2} \cap b_{6}\right)
$$

The outputs of OVSF code generator of $\mathrm{C}_{8,5}$ and $\mathrm{C}_{8,7}$ for $\mathrm{N}=5$ and $\mathrm{N}=7$ respectively are depicted together in Figure 9. 
Tamer A. Moniem and Noha EL Mosilhy, All optical OVSF code generator realization using ...
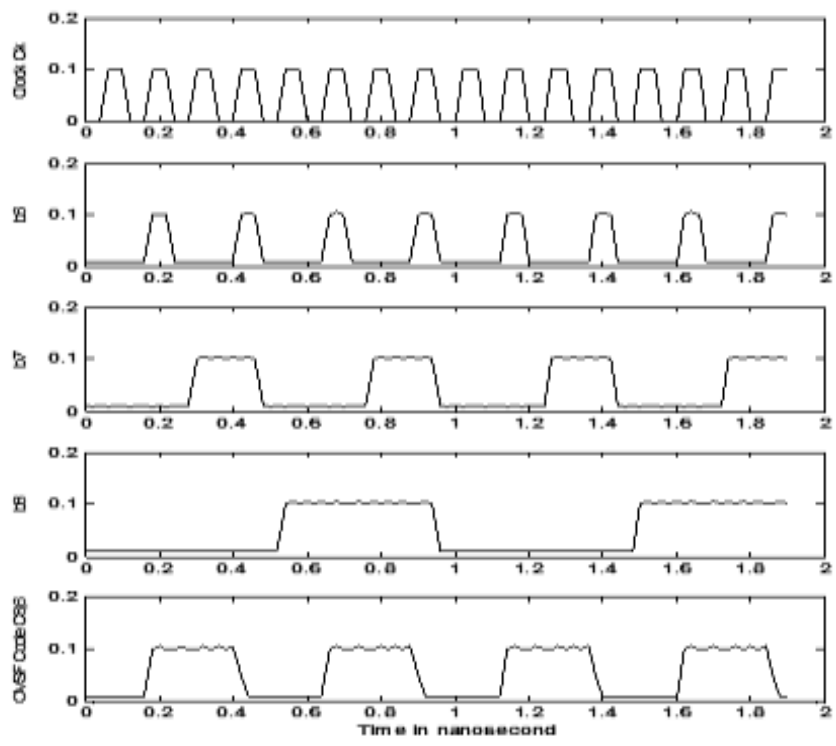

Fig. 8. The numerical simulation output code $\mathrm{C}_{8,6}$ at $\mathrm{N}=0110$ with $S F=8$ and counter range $b_{8} b_{7} b_{6}(0-8)$.
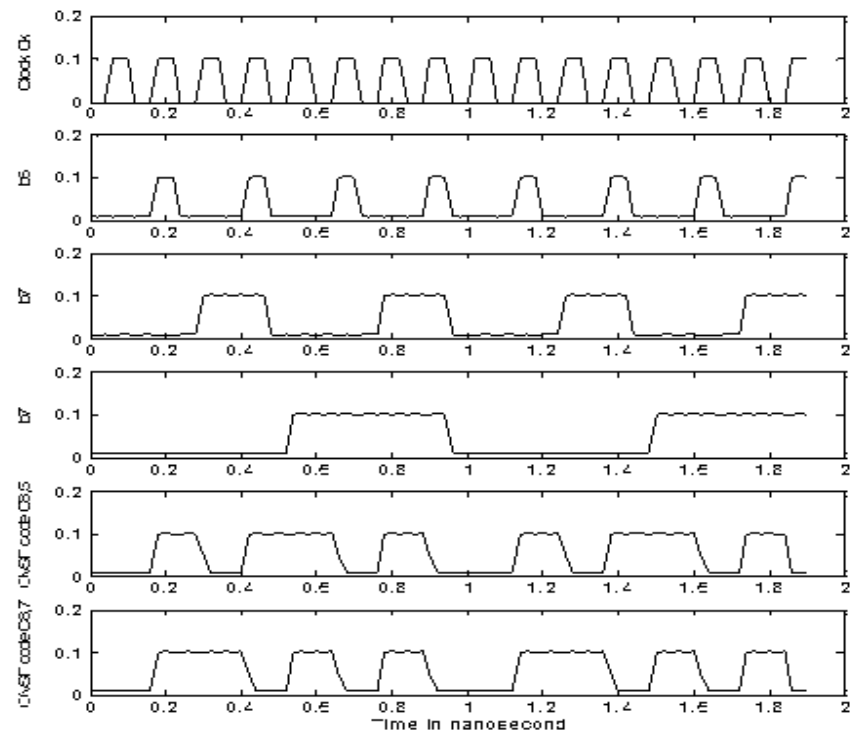

Fig. 9. The numerical simulation output codes $\mathrm{C}_{8,5}$ and $\mathrm{C}_{8,7}$ at $\mathrm{N}=0101$ and $\mathrm{N}=0111$ respectively.

\section{Conclusion}

In this paper, we have proposed and described all-optical schemes for OVSF code generator of spreading factor range $S F=4-512$. The generator consists of a series of optical $\mathrm{T}$ flip flops based on two coupled polarization switches, and a set of optical logic gates. Numerical simulation results confirming the described method are given. The theoretical model is developed and the results obtained experimentally backed by the numerically simulation result will be useful in the future for using the OVSF code tree techniques in all-optical computing and optical information processing to supports a lot of users in the optical communication links. 


\section{REFERENCES}

[1] E. Dahlman and K. Jamal, "Wide-Band Services in a DS-CDMA Based FPLMTS System", Proc. IEEE Vehicular Technology Conf. 1996, pp. 1656-1660, (1996).

[2] T.A. Moniem, N.A. Rabou, E.M. Saad, "Parallel-shift register and binary multiplier using optical hardware components", Opt. Eng. Vol 47, doi: 10.1117/1.2898632, (2008).

[3] Tanay Chattopadhyay, T. A. Moniem, Hirak Kumar Maity "All-Optical Pseudorandom Binary Sequence (PRBS) Generator Using The Hardlimiters”, OPTIK Elsevier journal 124, pp. 4252-4256, (2013).

[4] K.E. Zoiros, M.K. Das, D.K. Gayen, H.K. Maity, T. Chattopadhyay, J.N. Roy, "All-optical pseudorandom binary sequence generator with TOAD-based D flipflops", Opt. Commun. vol 284,pp. 4297-4306, (2011).

[5] Y.C. Tseng and C.M. Chao, "Code Placement and Replacement Strategies for Wideband CDMA OVSF Code Tree Management”, IEEE Trans. Mobile Computing, vol. 1, no. 4, pp. 293-302, ( 2002).

[6]Yuh-Shyan Chen, and Ting-Lung Lin, "Code Placement and Replacement Schemes for WCDMA RotatedOVSF Code Tree Management”, IEEE Trans. Mobile Computing, Vol. 5, no. 3, pp. 224-239, (2006).

[7] F. Adachi, M. Sawahashi, and H. Suda, "Wideband DS-CDMA for Next-Generation Mobile Communications Systems”, IEEE Comm. , vol. 36, pp. 56-69, (1998).

[8] Yu-Chee Tseng, Member, and Chih-Min Chao, "Code Placement and Replacement Strategies for Wideband CDMA OVSF Code Tree Management", IEEE Trans. Mobile Computing, vol. 1, no. 4, pp 293-302, (2002).

[9] R.G. Cheng and P. Lin," OVSF Code Channel Assignment for IMT-2000”, Proc. IEEE Vehicular Technology Conf., vol. 3, pp. 2188- 2192, (2000).

[10]W.T. Chen, Y.P. Wu, and H.C. Hsiao, "A Novel Code Assignment Scheme for W-CDMA Systems", Proc. IEEE Vehicular Technology Conf., vol. 2, 1182-1186, (2001).

[11] Dong H. Lee, 'Logic design of orthogonal variable spreading factor code generator", J. Circuit, Systems and Computers Vol. 14, pp. 507-513, (2005).

[12] Boris D. Andreev, Edward L. Titlebaum, and Eby G. Friedman, "Orthogonal Code Generator for 3G Wireless Transceivers”, GLSVLSI'03, Washington DC- USA April 28-29,(2003).

[13] T.A. Moniem , M.H. Saleh, "Fuzzy logic membership implementation using optical hardware components", Optics Communications vol 285, pp. 4474-4482, (2012).

[14]Y. Liu, M.T. Hill, H. de Waardt, G.D. Khoe, D. Lenstra, and H.J.S. Dorren, "All optical flipflop memory based on two polari-zation switches", Electron. Lett. Vo 38,pp. 904-905, (2002).

[15] Tamer A. Moniem," All optical Asynchronous Binary Counter Based on All Optical J-K flip-flop Memory", Proceeding of ICECECE 2011 conference- Bangkok, Thailand Dec 25-26, (2011).

[16] M.Morris Mano, "Computer engineering hardware design", prantice Hall international $3{ }^{\text {rd }}$ edition.

[17]G.P. Agrawal and N.K.Dutta, "Semiconductor lasers", Van Nostrand Reinhold, (1993). 
Tamer A. Moniem and Noha EL Mosilhy, All optical OVSF code generator realization using ...

تصميم مولا ضوئي كودي لمعامل الأتثنار متعامد التغير

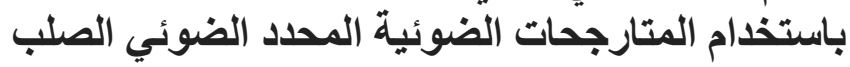

الملخص العربى

معامل الأنتشار متعامد التغير (OVSF) يقوم بتقديم معدلات متغيرة للبيانات ويدعم العديد من التطبيقات

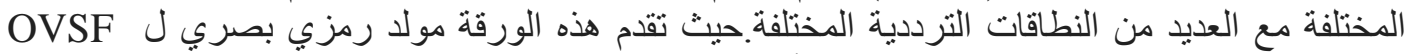

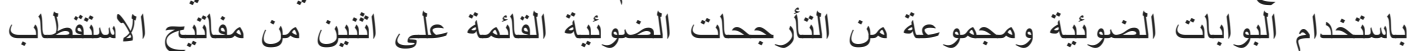

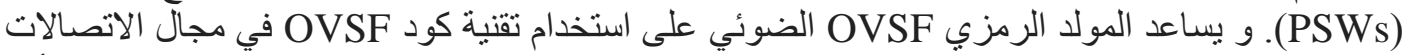

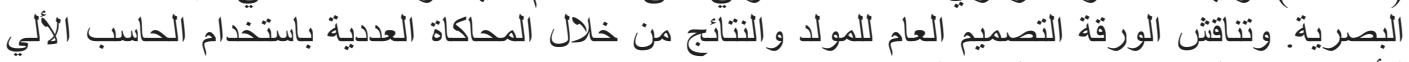
لتأكيد جدوى التصميم من خلال عاملي الانتشار 4 و 8 . 8. 